Fundación

Miguel Lillo

Tucumán

Argentina

\title{
Primer registro de homing en el peludo Chaetophractus villosus (Xenarthra: Chlamyphoridae)
}

\author{
First record of homing in the large hairy armadillo Chaetophractus villosus \\ (Xenarthra: Chlamyphoridae)
}

Jorge A. Gallo ${ }^{1,2 *}$, Juan I. Reppucci ${ }^{3}$, Laura Fasola 1,2, Agustín Ayuso4, Agustín Manuel Abba 5

1 Consejo Nacional de Investigaciones Científicas y Técnicas (CONICET), Dirección Regional Patagonia Norte, Administración de Parques Nacionales. Vice Almte. O'Connor 1188, (8400) San Carlos de Bariloche, Río Negro, Argentina. [Correspondencia: jorgegallo2110@gmail.com]

2 Programa Patagonia - Aves Argentinas. Matheu 1246, (1249) Ciudad Autónoma de Buenos Aires.

3 Consejo Nacional de Investigaciones Científicas y Técnicas (CONICET), Dirección Regional Noroeste, Administración de Parques Nacionales. Santa Fe 23, (4400) Salta, Argentina.

4 Conservación Península Valdés, Estancia Rincón Chico.

5 Centro de Estudios Parasitológicos y de Vectores (CEPAVE), Universidad Nacional de La Plata - CONICET. Boulevard 120 S/N entre Avenida 60 y calle 64, (1900) La Plata, Buenos Aires, Argentina.

\section{RESUMEN}

Se registró por primera vez el comportamiento de homing en Chaetophractus villosus. Se capturaron 33 individuos de $C$. villosus y se liberaron a diferentes distancias en Península Valdés, Chubut, Argentina. Del total de individuos, el 30 \% regresó al punto captura dentro de los 12 días desde la liberación. Se observó que la correlación entre la distancia y los días hasta la recaptura fue negativa y significativa. Aunque son necesarios nuevos estudios, el regreso al sitio de captura posiblemente se debe a que los peludos reconozcan a este sitio como fuente de recursos alimenticios.

Palabras clave - Argentina, armadillo, Chubut, Patagonia, Península Valdés, translocación.

\section{ABSTRACT}

We registered the first record of homing behavior in Chatophractus villosus. Thirtythree individuals of $C$. villosus were captured and released at different distances

Ref. bibliográfica: Juárez, M. 2021. "Sobre-veraneo de Calidris canutus rufa (Charadriiformes: Scolopacidae) en laguna La Picasa, Santa Fe, Argentina". Acta zoológica lilloana 65 (2): 261-267. doi: https://doi. org/10.30550/j.azl/2021.65.2/2021-11-03

- Recibido: 3 de septiembre 2021 - Aceptado: 3 de noviembre 2021.

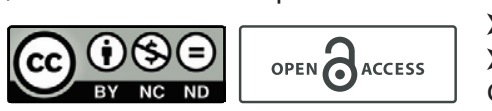

- URL de la revista: http://actazoologica.lillo.org.ar

- Esta obra está bajo una Licencia Creative Commons Atribución - No 
in PenínsulaValdés, Chubut, Argentina. Of the total number of individuals, 30\% returned to the point of capture within 12 days. The correlation between distance and days until recapture was negative and significant. Although further studies are necessary, is possibly that the capture site was recognized by the large hairy armadillo as a source of food resources.

Keywords - Argentina, armadillo, Chubut, Patagonia, Península Valdés, translocation.

La translocación de fauna ha sido utilizada como herramienta de manejo durante mucho tiempo, ya sea para la conservación de especies en peligro o para mitigar posibles impactos sobre actividades antrópicas (Massei, Quy, Gurney y Cowan, 2010; Cannella y Henry, 2016). Sin embargo, el éxito de la translocación de especies depende de muchos factores, como ser el estrés al que son sometidos los individuos, las características del sitio de liberación y el comportamiento conocido como homing. Éste es un comportamiento instintivo de la fauna caracterizado por una tendencia a regresar al sitio de captura luego de un evento de translocación (Yahner, 2012). Aunque ocurre frecuentemente en grandes mamíferos, se han registrados casos de homing en pequeños y medianos mamíferos (Villaseñor, Escobar y Estades, 2013).

En armadillos se registraron eventos de homing solo para Dasypus novemcinctus. Para esta especie, algunos estudios encontraron que los individuos liberados a distancias dentro de su área de acción, regresan al sitio de captura (Layne y Glover, 1977; Chamberlain, 1980; Gammons, Mengak y Conner, 2009). Si bien el motivo del regreso luego de la translocación no está bien estudiado, Gammons et al. (2009) aseguran que $D$. novemcinctus es capaz de reconocer rastros odoríferos producidos en las glándulas perianales de estos armadillos.

El peludo Chaetophractus villosus (Xenarthra: Cingulata) es una de las 14 especies de armadillos presentes en Argentina (SAyDS-SAREM, 2019). Se distribuye desde el Gran Chaco boliviano, Paraguay, hasta la Provincia de Santa Cruz en Argentina (Gallo, et al., 2019). En Argentina fue introducido en Patagonia en Isla Leones (Chubut) y en la Isla Grande de Tierra del Fuego (Poljak, Escobar, Deferrari y Lizarralde, 2007; Gallo et al., 2019). Se encuentra en una gran variedad de hábitats, incluyendo pastizales, la estepa y áreas degradadas como ser agroecosistemas (Abba y Cassini, 2008; Abba, Tognelli, Seitz, Bender y Vizcaíno, 2012; Abba, Zufiaurre, Codesido y Bilenca, 2016). La presencia de este armadillo está asociada a diversas actividades antrópicas como campos agrícola-ganaderos, basurales y compostajes domiciliarios; estas actividades facilitan el acceso a recursos alimenticios y le permiten incrementar su actividad y dispersión (Abba, Poljak, Gabrielli, Teta y Pardiñas, 2014; Abba, Zufiaurre, Codesido y Bilenca, 2015; Abba, Gallo y Zufiaurre, 2018).

Entre los meses de marzo y junio de 2018 fueron capturados 33 individuos de $C$. villosus en el casco de la Estancia Rincón Chico, Península Valdés, Chubut, Argentina. La zona pertenece a la ecorregión Estepa Patagónica y se caracteriza por un clima frio, ventoso y seco. La vegetación predominante corresponde a matorrales xerófilos achaparrados y herbáceas (Figura 1; Matteucci, 2011). 


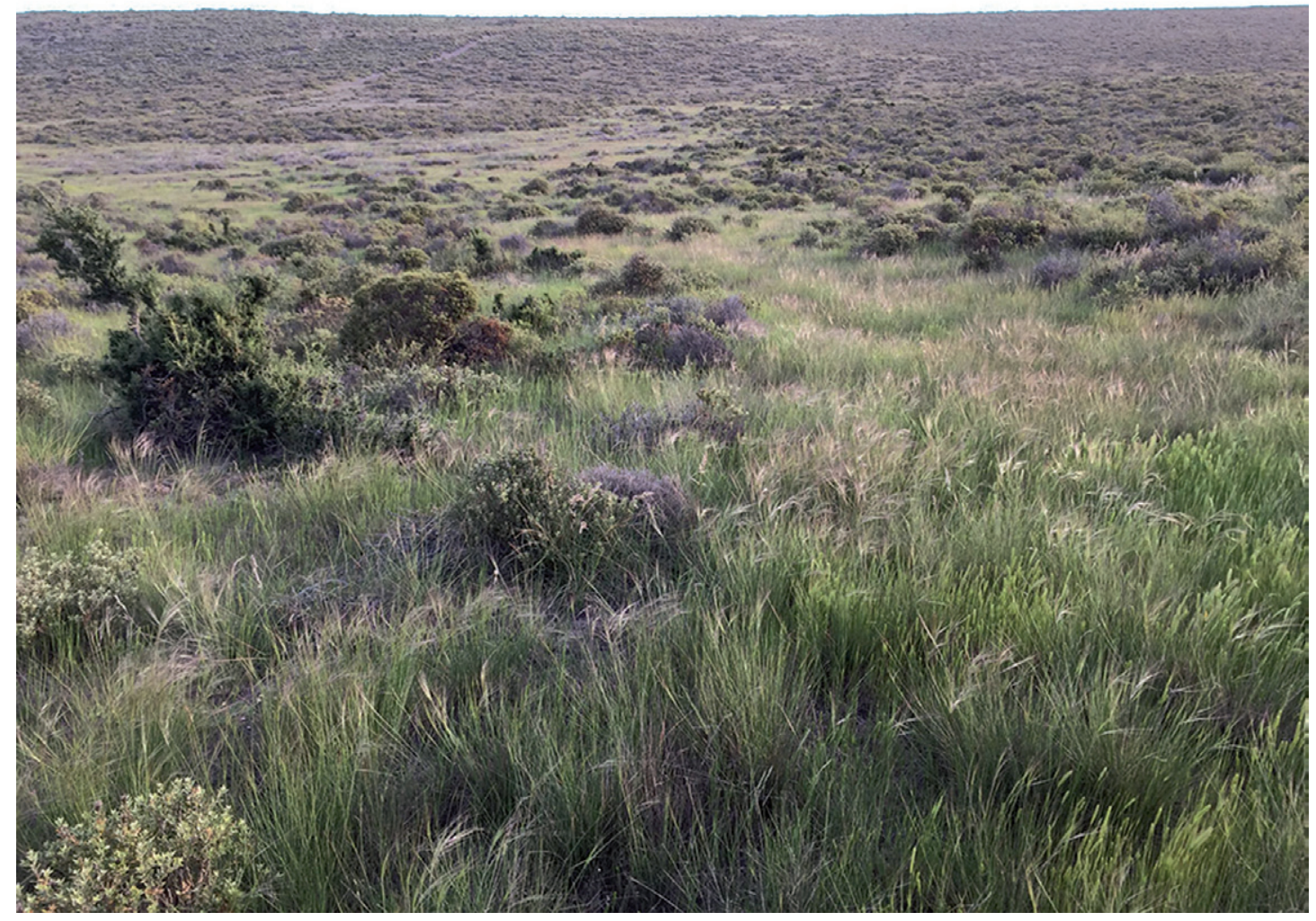

Figura 1. Ambiente perteneciente al área de estudio en la Ea. Rincón Chico, Península Valdés, Chubut, Argentina. Se observan matorrales xerófilos y herbaceas, vegetación típica de la ecorregión Estepa Patagónica.

Figure 1. Study area environment at Ea. Rincon Chico, Peninsula Valdes, Chubut, Argentina. Xerophilous and herbaceous bushes are observed, typical vegetation of the Patagonian steppe ecoregion.

Los armadillos fueron marcados y liberados a distintas distancias lineales desde el casco de la estancia: $3500 \mathrm{~m} ; 4100 \mathrm{~m} ; 4400 \mathrm{~m} ; 5000 \mathrm{~m} ; 5200 \mathrm{~m}$ y $5500 \mathrm{~m}$ (Figura 2). Se registró el día de la liberación y el día en que se observó nuevamente al individuo en el casco de la estancia. Las detecciones de los armadillos fueron a través de cámaras trampas colocadas en el casco de la estancia y por observación directa de los individuos. Para evaluar si había una asociación entre la distancia de liberación y la probabilidad que los individuos regresaran al origen o no, se utilizó un modelo lineal generalizado con distribución del error de tipo binomial. Luego, se usó una correlación de Pearson entre la distancia de liberación y el tiempo de regreso para los individuos que regresaron al origen. Ambos análisis se realizaron usando $R(R$ Core Team, 2019).

De los 33 individuos capturados en solo 10 se observó comportamiento de homing (30\%). El tiempo mínimo de recaptura fue de 1 día para $5500 \mathrm{~m}(\mathrm{n}=1)$ y el máximo fue de 12 días para $3500 \mathrm{~m}(\mathrm{n}=1$; Figura 3). La probabilidad de regreso/ recaptura no estuvo asociada a la distancia de liberación $(Z=0,345 ; p=0,73)$. Para los individuos que regresaron al punto de origen, se vio que la correlación entre la distancia y los días hasta la recaptura fue negativa y significativa $(\mathrm{r}=-0,72$; g.l. $=8$, $\mathrm{p}=0,018)$. 


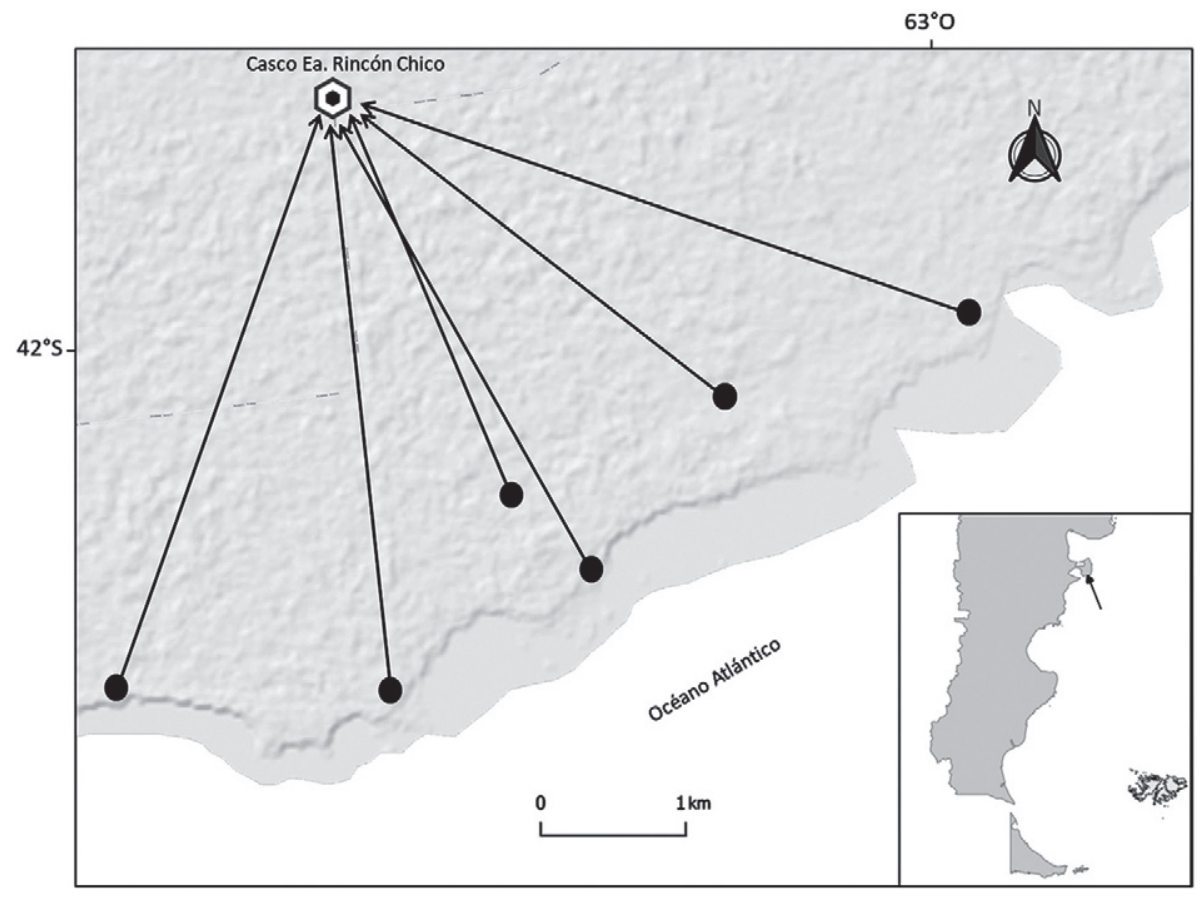

Figura 2. Mapa del área de estudio en la Ea. Rincón Chico, Penisnula Valdés, Chubut. Los círculos negros indican los sitios de liberación de Cheatophractus villosus. Las flechas apuntan hacia el sitio de recaptura donde se registró el comportamiento de homing en algunos individuos.

Figure 2. Map of the study area in Ea. Rincón Chico, Península Valdés, Chubut. Black circles indicate Cheatophractus villosus release sites. The arrows point out the recapture site where homing behavior was registered in some individuals.

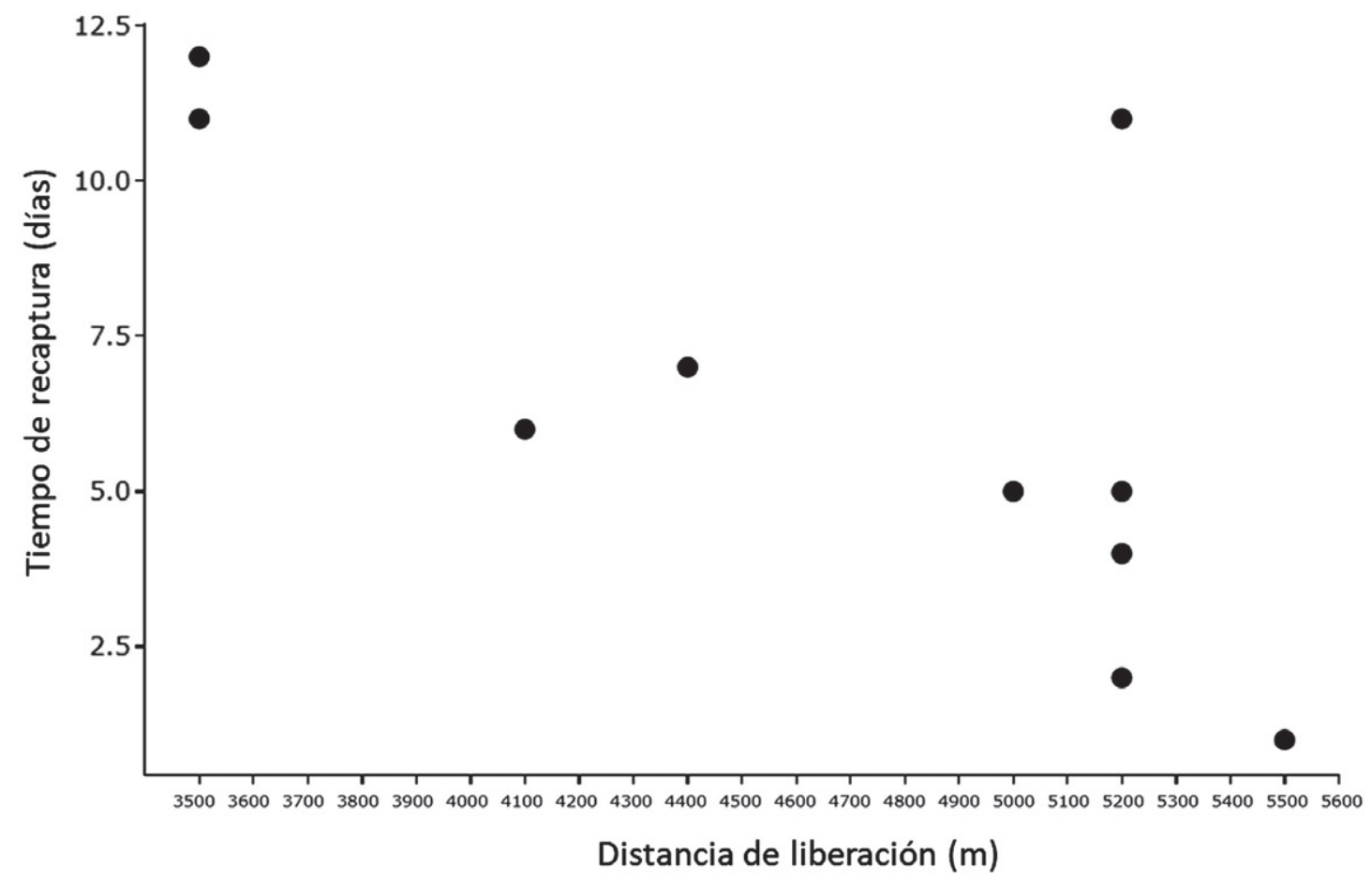

Figura 3. Gráfico de puntos mostrando la relación entre la distancia de translocación (metros) y el tiempo transcurrido hasta la recaptura (días).

Figure 3. Dot plot showing the relationship between translocation distance (meters) and recapture time (days). 
Se ha encontrado que ciertos mamíferos son capaces de explorar sitios por fuera de su área de acción y reconocer el ambiente circundante logrando orientarse en el espacio (Powell y Mitchell, 2012; Villaseñor, et al. 2013). En este caso, no todas las translocaciones resultaron en la recaptura del animal en el punto de origen y la probabilidad de que esto ocurra no estuvo asociada a la distancia al punto de liberación. Esta falta de relación puede deberse a que algunos de los individuos fueron liberados en puntos demasiado alejados de su área de acción tal que no han podido orientarse y regresar. También, considerando el comportamiento generalista-oportunista del peludo, podría pensarse, que ciertos individuos pudieron localizar otra fuente de recursos más cercana al punto de liberación.

Finalmente, la correlación entre la distancia y el tiempo transcurrido hasta la recaptura (en aquellos que regresaron) podría indicar que el casco de la Estancia Rincón Chico es una fuente importante de recursos alimenticios para el peludo (ver Abba et al. 2018) que es compartida entre varios individuos y además, no habría barreras reconocibles para el desplazamiento de estos individuos en el espacio entre el origen y los puntos de liberación.

Sin embargo, considerando que en Patagonia se encuentran dos poblaciones invasoras de C. villosus, este primer registro de homing en peludos resulta de especial interés para diseñar planes de manejo. Así mismo, para asegurar la eficacia de estos planes, es necesario profundizar en estudios que permitan conocer el área de acción real de este armadillo y estudiar cuales son las variables ambientales y espaciales que permiten la su orientación en el espacio. Como así también nuevos estudios asociados a la disponibilidad de recursos en el sitio de captura que permitan explicar el retorno de los armadillos.

\section{AGRADECIMIENTOS}

A Conservación Península Valdés y Estancia Rincón Chico por el financiamiento y apoyo logístico. A Kini Roesler por sus aportes a la discusión.

\section{FINANCIAMIENTO}

Conservación Península Valdés y Estancia Rincón Chico.

\section{PARTICIPACIÓN}

J. R. y A. A. diseñaron el experimento y realizaron la toma de datos. J. A. G., A. M. A. y L. F. realizaron los modelos y análisis estádisticos. J. A. G. escribió la primer versión del manuscrito. Todos los autores contribuyeron a la versión final del manuscrito. 


\section{LITERATURA CITADA}

Abba, A. M., Zufiaurre, E., Codesido, M., Bilenca, D. N. (2015). Burrowing activity by armadillos in agroecosystems of central Argentina: Biogeography, land use, and rainfall effects. Agriculture, Ecosystems and Environment, 200, 54-61. https://doi.org/10.1016/j.agee.2014.11.001

Abba, A. M., Cassini, M. H. (2008). Ecology and conservation of three species of armadillos in the Pampas region, Argentina. En The Biology of the Xenarthra (300-305). Gainesville: University Press of Florida.

Abba, A. M., Zufiaurre, E., Codesido, M., Bilenca, A. D. N. (2016). Habitat use by armadillos in agroecosystems of central Argentina: does plot identity matter? Journal of Mammalogy, 20, 1-7. https://doi.org/10.1093/jmammal/gyw100

Abba, A. M., Gallo, J. A., Zufiaurre, E. (2018). Uso de basurales por parte del peludo (Chaetophractus villosus). Notas Sobre Mamíferos Sudamericanos, 01, 001-005. https://doi.org/10.31687/saremNMS.19.0.02

Abba, A. M., Tognelli, M. F., Seitz, V. P., Bender, J. B., Vizcaíno, S. F. (2012). Distribution of extant xenarthrans (Mammalia: Xenarthra) in Argentina using species distribution models. Mammalia, 76, 123-136. https://doi.org/10.1515/mammalia-2011-0089

Abba, A. M., Poljak, S., Gabrielli, M., Teta, P., Pardiñas, U. F. J. (2014). Armored invaders in patagonia: recent southward dispersion of armadillos (CINGULATA, DASYPODIDAE). Mastozoologia Neotropical, 21, 311-318.

Cannella, E. G., Henry, J. (2016). A case of homing after translocation of chuditch, Dasyurus geoffroii (Marsupialia/ : Dasyuridae). Australian Mammalogy, 38, 118-120. https://doi.org/10.1071/WR08179

Chamberlain, P. A. (1980). Armadillos: Problems and control. In Proceedings of the 9th Vertebrate Pest Conference.

Gallo, J. A., et al. (2019). Chaetophractus villosus. En SAyDS-SAREM (Ed.), Categorización 2019 de los mamíferos de Argentina según su riesgo de extinción. Lista Roja de los mamíferos de Argentina. www.cma.sarem.org.ar

Gammons, D., Mengak, M. T., Conner, L. M. (2009). Translocation of nine-banded armadillos. Human-Wildlife Confl Icts, 3, 64-71.

Layne, J. N., Glover, D. (1977). Home range of the armadillo in Florida. Journal of Mammalogy, 58, 411-413. https://doi.org/10.2307/1379340

Massei, G., Quy, R. J., Gurney, J., \& Cowan, D. P. (2010). Can translocations be used to mitigate humanwildlife conflicts? Wildlife Research, 37, 428-439. https://doi. org/10.1071/WR08179

Matteucci, S. D. (2011). Ecorregión Estepa Patagónica. En J. Morello, S. D. Matteucci, A. F. Rodríguez, \& M. Silva (Eds.), Ecorregiones y complejos ecosistémicos argentinos.

Poljak, S., Escobar, J., Deferrari, G., Lizarralde, M. (2007). Un nuevo mamífero introducido en la Tierra del Fuego: El "peludo" Chaetophractus villosus (Mammalia, Dasypodidae) en Isla Grande. Revista Chilena de Historia Natural, 80, 285-294. https://doi.org/10.4067/S0716-078X2007000300003 
Villaseñor, N. R., Escobar, M. A. H., Estades, C. F. (2013). There is no place like home: High homing rate and increased mortality after translocation of a sma11 mammal. European Journal of Wildlife Research, 59, 749-760. https://doi. org/10.1007/s10344-013-0730-y

Yahner, R. H. (2012). Wildlife behavior and conservation. Wildlife Behavior and Conservation, 1-173. https://doi.org/10.1007/978-1-4614-1518-3 